\title{
Determinação do Módulo de Elasticidade de Painéis Aglomerados por Stress Wave Timer
}

\author{
Rafael Farinassi Mendes ${ }^{1}$, Lourival Marin Mendes ${ }^{1}$, \\ Amélia Guimarães Carvalho' ${ }^{1}$, José Benedito Guimarães Júnior ${ }^{2}$, \\ Ricardo Gabriel de Almeida Mesquita ${ }^{1}$ \\ ${ }^{1}$ Departamento de Ciências Florestais, Universidade Federal de Lavras - UFLA \\ ${ }^{2}$ Universidade Federal do Piauí - UFPI
}

\begin{abstract}
RESUMO
O objetivo do trabalho foi avaliar a determinação do módulo de elasticidade de painéis aglomerados por stress wave timer, assim como associar os resultados com o efeito de algumas variáveis de produção dos painéis. O delineamento experimental constituiuse em cinco tratamentos, sendo quatro tratamentos produzidos com bagaço de cana, nos quais foram avaliados dois tipos de adesivos (melamina-ureia-formaldeído - MUF e ureiaformaldeído - UF) em associação com dois tipos de estruturas de painel (em camadas e homogêneos) e um tratamento produzido com o adesivo UF, com estrutura em camadas e madeira de Eucalyptus urophylla. Observou-se que o tipo de material é o que exerce maior influência sobre a determinação do MOEd, sendo o painel produzido com bagaço de cana o que apresentou as melhores correlações. $\mathrm{O}$ tipo de adesivo não apresentou influência sobre os resultados, enquanto que os painéis com estrutura em camadas tiveram tendência de melhor correlação em relação aos painéis homogêneos.
\end{abstract}

Palavras-chave: variáveis de produção, bagaço de cana e eucalipto.

\section{Determination of the Elastic Modulus of Particleboard by Stress Wave Timer}

\begin{abstract}
The objective of this study was to evaluate the determination of the elastic modulus of particleboard by stress wave timer and associate the results with the effect of some production variables of the panels. The experiment consisted in five treatments: four treatments produced with sugar cane bagasse, where two types of adhesives (melamine-urea-formaldehyde - MUF and urea-formaldehyde - UF) were evaluated in combination with two types of panel structures (layered and homogeneous); and one treatment produced with UF adhesive, with structure in layers and with Eucalyptus urophylla wood. It was possible to observe that the type of material is what influences the most on the determination of MOEd, with the panel of sugar cane bagasse presenting the best correlations. The type of adhesive had no effect on the results, while the panels with layered structure tended to have better correlation in relation to the homogeneous panels.
\end{abstract}

Keywords: variable production, sugar cane bagasse and eucalyptus. 


\section{INTRODUÇÃO}

Os setores de construção civil e de mobiliário são os principais responsáveis pela evolução tecnológica do segmento das indústrias de painéis aglomerados, tendo em vista a utilização destes como matériaprima básica.

Atualmente, tal segmento utiliza basicamente a madeira de pinus e eucalipto. No entanto, várias alternativas estão sendo estudadas para a produção desses painéis, dentre as quais, o bagaço de cana é o que tem obtido maior destaque, devido principalmente à quantidade produzida, pois, de acordo com dados do IBGE (2010), no Brasil, a produção anual de bagaço de cana está em torno de 195 milhões de $t$ (Mendes et al., 2010) e também em função das boas propriedades físico-mecânicas dos painéis produzidos (Mendes et al., 2008, 2009, 2010).

Para a análise das propriedades físico-mecânicas dos painéis aglomerados, tradicionalmente são realizados ensaios destrutivos, os quais são demorados e trabalhosos, além de que exige um grande número de amostras.

No entanto, novas técnicas, como os métodos não destrutivos estão sendo avaliados para a avaliação das propriedades da madeira e de seus subprodutos. Dentre eles, podem ser destacados os testes de stress wave, ultrassom, deflexão, propriedades elétricas, radiações gama, espectroscopia no infravermelho próximo e método de raios X (Morales, 2006).

$\mathrm{Na}$ técnica do stress wave, são geradas ondas através de uma vibração produzida por impacto na peça em estudo. Mede-se a velocidade do som, cujo valor é utilizado na determinação da constante dinâmica (Bodig, 2001). O stress wave utiliza baixos movimentos moleculares de tensão para medir duas propriedades fundamentais dos materiais: a energia armazenada e a dissipação. A energia armazenada manifesta-se pela velocidade com a qual a onda percorre o material, enquanto que a taxa sob a qual a onda é atenuada é uma indicação de dissipação de energia (Morales, 2006).

Alguns trabalhos já foram desenvolvidos com painéis de madeira, sendo demonstrada forte correlação entre o módulo de elasticidade dinâmico principalmente com o módulo de elasticidade estático obtido por teste destrutivo em ensaio de flexão (Vun et al., 2004; Morales, 2006; Del Menezzi et al., 2007), sendo demonstrado ainda que o stress wave pode ser adequadamente utilizado para a predição das propriedades de painéis de madeira.

Neste contexto, o objetivo do trabalho foi avaliar a correlação entre o módulo de elasticidade dinâmico de painéis aglomerados, obtido por método não destrutivo (stress wave timer), com o módulo de elasticidade obtido por flexão estática, assim como associar os resultados com o efeito de algumas variáveis de produção dos painéis.

\section{MATERIAL E MÉTODOS}

O bagaço de cana foi coletado na Usina Monte Alegre, na cidade de Areado - MG. Enquanto que a madeira de Eucalyptus urophylla com oito anos de idade foi obtida em um plantio na cidade de Paracatu-MG.

O delineamento experimental se constituiu de cinco tratamentos, conforme destacado na Tabela 1.

Depois da coleta do bagaço de cana e da madeira de Eucalyptus urophylla, estes foram triturados em um moinho martelo, para a geração das partículas do tipo sliver, com granulometria de $1,72 \mathrm{e}$ $6,14 \mathrm{~mm}$, as quais foram usadas na face e no miolo, respectivamente, de acordo com o delineamento experimental apresentado na Tabela 1. Os painéis homogêneos foram produzidos apenas com as partículas de $6,14 \mathrm{~mm}$.

Tabela 1. Delineamento experimental.

Table 1. Experimental design.

\begin{tabular}{c|lcc|}
\hline Tratamentos & Matéria-prima & Adesivo & Composição \\
\hline T1 & Bagaço de cana & UF & Homogêneo \\
T2 & Bagaço de cana & & Camadas \\
T3 & Bagaço de cana & MUF & Homogêneo \\
T4 & Bagaço de cana & UF & Camadas \\
T5 & Eucalyptus urophylla & & Camadas \\
\hline
\end{tabular}


As partículas foram então secas a um teor de umidade de aproximadamente $3 \%$ e posteriormente levadas para uma encoladeira do tipo tambor giratório, onde foi aplicado $9 \%$ de adesivo e ainda $1 \%$ de parafina para todos os tratamentos.

Depois de encoladas, as partículas foram depositadas em uma caixa formadora de colchão, com dimensões de $48 \times 48 \mathrm{~cm}$, para a realização da pré-prensagem, a qual ocorreu em uma prensa manual a $4 \mathrm{kgf} / \mathrm{cm}^{2}$. Posteriormente, o colchão foi levado para uma prensagem a quente, onde permaneceu por um período de 8 minutos, a uma temperatura de $160^{\circ} \mathrm{C}$ e pressão de $40 \mathrm{kgf.cm}{ }^{-2}$. Para cada tratamento, foram produzidos quatro painéis com densidade nominal de 0,70 g.cm ${ }^{-3}$.

Em cada um dos painéis, foram realizadas três leituras com o equipamento stress wave timer, e foi obtido o tempo de propagação de onda entre os dois transductos do equipamento, os quais foram posicionados em cada uma das bordas dos painéis. O módulo de elasticidade dinâmico foi determinado conforme as Equações 1 e 2.

$\operatorname{MOEd}=\mathrm{d} \times \mathrm{V}^{2} \times 1 / \mathrm{g}$

$\mathrm{V}=\mathrm{D} / \mathrm{t}$

em que: MOEd = Módulo de elasticidade dinâmico $\left(\mathrm{Kgf} / \mathrm{cm}^{2}\right)$; d = Massa específica do painel $\left(\mathrm{Kg} / \mathrm{m}^{3}\right) ; \mathrm{g}=$ Aceleração da gravidade $\left(9,804 \mathrm{~m} / \mathrm{s}^{2}\right) ; \mathrm{V}=$ Velocidade da onda longitudinal $(\mathrm{cm} / \mathrm{s}) ; \mathrm{V}=$ Velocidade $(\mathrm{cm} / \mathrm{s}) ; \mathrm{D}$ = distância entre os transdutores $(\mathrm{cm}) ; \mathrm{t}=$ tempo de propagação $(\mathrm{s})$.

Os testes destrutivos para o módulo de elasticidade (MOE) à flexão foram determinados conforme a norma DIN 52362 (DIN, 1982).

Para a avaliação dos dados, foram realizadas análise de regressão linear e teste de correlação de Pearson, ambos a 1 e 5\% de significância.

\section{RESULTADOS E DISCUSSÃO}

$\mathrm{Na}$ Tabela 2, encontram-se os valores de correlação de Pearson entre o módulo de elasticidade dinâmico, obtido pelo método não destrutivo, e o módulo de elasticidade à flexão estática para cada uma das variáveis de produção dos painéis.
Tabela 2. Correlação de Pearson entre o MOEd e MOE. Table 2. Pearson correlation between the MOEd and MOE.

\begin{tabular}{lc} 
Tipo de adesivos & Correlação entre MOED e MOE \\
\hline UF & $0,9516^{\star *}$ \\
MUF & $0,9681^{\star *}$ \\
Camadas & $0,9863^{\star *}$ \\
Homogêneo & $0,9231^{\star *}$ \\
Bagaço de cana & $0,9598^{\star}$ \\
Eucalipto & $0,7704^{\star}$
\end{tabular}

** significativo a $1 \%$; ${ }^{*}$ significativo a $5 \%$.

Observa-se que o índice de correlação para os tipos de adesivos aplicados aos painéis produzidos com o bagaço de cana não apresentaram grande influência sobre a correlação entre o MOEd e MOE.

Para o tipo de constituição do painel (camadas ou homogêneo), os painéis em camadas tiveram a tendência de melhora dos valores de correlação, fato este que pode estar associado a uma maior compactação das faces dos painéis em camadas (MDP), visto que as dimensões das partículas são menores, o que pode ter permitido uma melhor propagação da onda emitida.

Em relação ao tipo de matéria-prima utilizada para a produção dos painéis, a correlação entre o MOEd e MOE dos painéis de bagaço de cana foram superiores à encontrada para os painéis de eucalipto. Tal fato pode estar associado à menor densidade básica do bagaço de cana $\left(0,09 \mathrm{~g} / \mathrm{cm}^{3}\right)$ em relação à madeira de eucalipto $\left(0,52 \mathrm{~g} / \mathrm{cm}^{3}\right)$, o que permite assim uma maior compactação das partículas e, consequentemente, a diminuição dos espaços vazios entre elas, o que permite uma melhor propagação da onda emitida pelo equipamento, e assim uma melhor correlação entre as propriedades MOEd e MOE.

Contudo, apesar da análise das correlações de Pearson, também foi verificada a análise de regressão para cada um dos tipos de variáveis de produção dos painéis aglomerados, com o intuito de avaliar a tendência das correlações.

\subsection{Efeito do tipo de adesivo}

Nas Figuras 1 e 2, estão demonstradas as correlações entre o MOE e o MOEd para os painéis de bagaço de cana produzidos com os adesivos UF e MUF, respectivamente. 


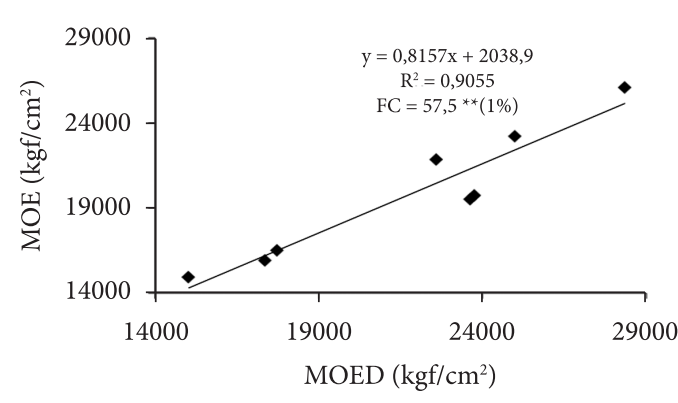

Figura 1. Correlação entre MOE e MOEd para os painéis com adesivo ureia-formaldeído.

Figure 1. Correlation between MOE and MOEd for panels with urea-formaldehyde adhesive.

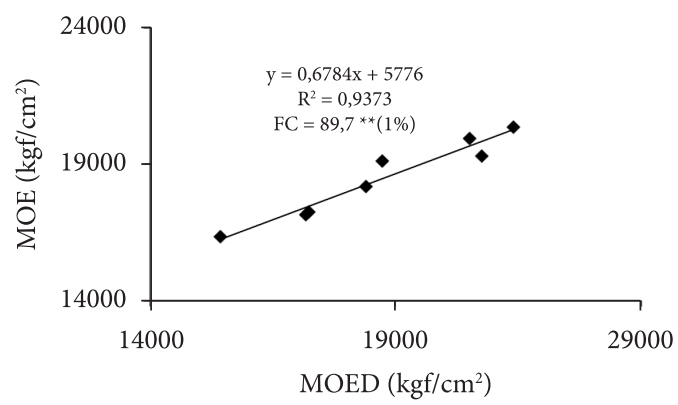

Figura 2. Correlação entre MOE e MOEd para os painéis com adesivo melamina-ureia-formaldeído.

Figure 2. Correlation between MOE and MOEd for panels with melamine-urea-formaldehyde adhesive.

Nos dois tipos de adesivos (ureiaformaldeído - UF; melamina-ureia-formaldeídoMUF), houve forte correlação entre o MOE e o MOEd, sendo os valores de $\mathrm{R}^{2}$ de 0,9055 e 0,9373, respectivamente. Em ambos os casos, a tendência de correlação dos dados foi linear.

Vun et al. (2004), avaliando diferentes variáveis de produção de painéis aglomerados de bagaço de cana, também não observaram efeito significativo do tipo de adesivo (fenol-formaldeído; ureia-formaldeído e dimetil-isocianato) sobre a determinação não destrutiva das propriedades mecâncias dos painéis.

\subsection{Efeito da composição dos painéis}

Nas Figuras 3 e 4, estão demonstradas as correlações entre o MOE e o MOED para os painéis de bagaço de cana produzidos de forma homogênea e em camadas, respectivamente.

No caso da avaliação do tipo de estrutura dos painéis aglomerados de bagaço de cana (homogêneo e camadas), a regressão linear se mostrou com

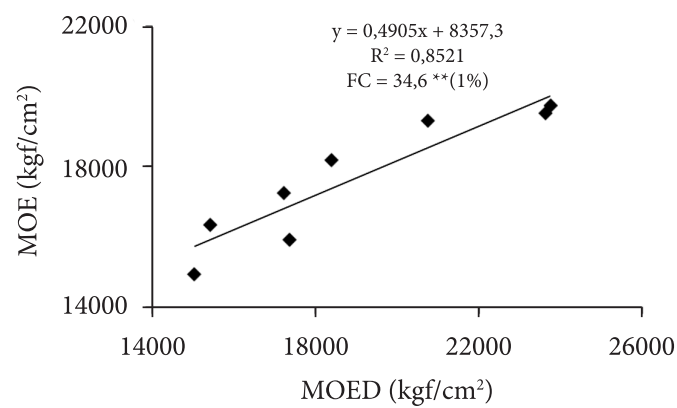

Figura 3. Correlação entre MOE e MOEd para os painéis homogêneos.

Figure 3. Correlation between MOE and MOEd for homogeneous panels.

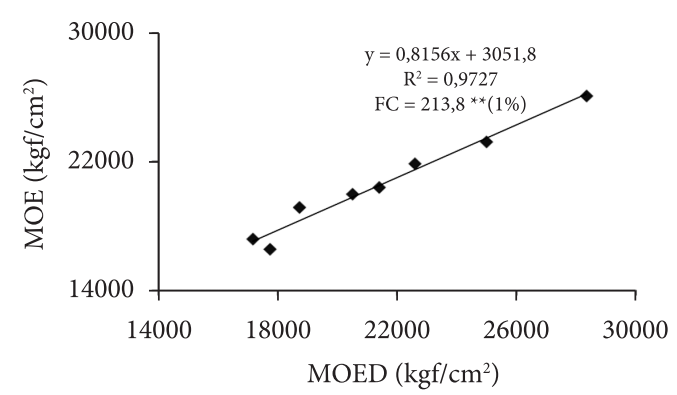

Figura 4. Correlação entre MOE e MOED para os painéis em camadas.

Figure 4. Correlation between MOE and MOED for panels in layers.

maiores valores de $\mathrm{R}^{2}$ para os painéis em camadas $\left(\mathrm{R}^{2}\right.$ de 0,9727$)$ do que o obtido pelos painéis homogêneos $\left(\mathrm{R}^{2}\right.$ de 0,8521$)$.

Vun et al. (2004) afirmam que o aumento da compactação dos painéis de aglomerados de bagaço de cana permite melhor propagação das ondas de tensão no material e assim pode resultar melhor relação entre o MOEd e MOE.

\subsection{Efeito da matéria-prima}

Nas Figuras 5 e 6, estão demonstradas as correlações entre o MOE e o MOED para os painéis de bagaço de cana e para os painéis de madeira de eucalipto, respectivamente.

O tipo de matéria-prima foi o que mostrou a maior diferenciação estatística, sendo que o bagaço de cana apresenta um alto valor de $\mathrm{R}^{2}(0,976)$, enquanto que os painéis produzidos com a madeira de eucalipto apresentaram um $\mathrm{R}^{2}$ inferior $(0,5936)$. Tal fato pode estar associado à maior razão de compactação dos painéis aglomerados de bagaço de cana, conforme 


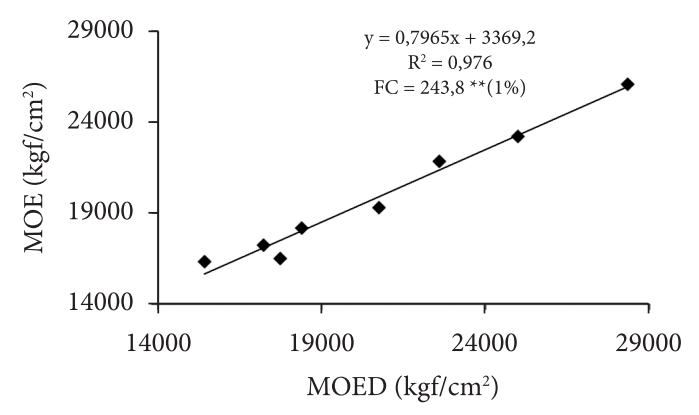

Figura 5. Correlação entre MOE e MOEd para os painéis de bagaço de cana.

Figure 5. Correlation between MOE and MOEd for the panels from sugarcane bagasse.

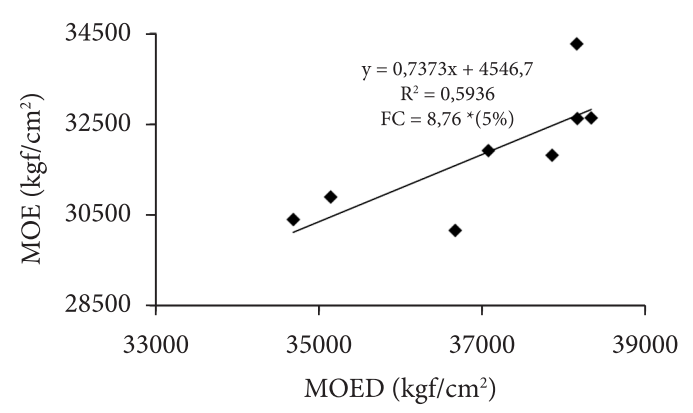

Figura 6. Correlação entre MOE e MOED para os painéis de madeira de eucalipto.

Figure 6. Correlation between MOE and MOED for panels of eucalyptus wood.

citado anteriormente. O mesmo foi verificado por Vun et al. (2004), os quais, avaliando diferentes variáveis de produção de painéis aglomerados de bagaço de cana e também painéis com a madeira da espécie Juniperus virginiana, obtiveram coeficiente de determinação $\left(\mathrm{R}^{2}\right)$ entre o MOEd (obtido por ultrassom) e o MOE (obtido por ensaio destrutivo) na faixa de 0,72 a 0,88 para os painéis de bagaço de cana e $\mathrm{R}^{2}$ na faixa de 0,50 a 0,75 para os painéis de Juniperus virginiana.

Morales (2006), avaliando em painéis OSB, observou coeficiente de determinação $\left(R^{2}\right)$, entre o MOED obtido por stress wave com o MOE obtido por ensaio destrutivo, variando de 0,32 a 0,61 . Enquanto que Del Menezzi et al. (2007) encontrou para painéis OSB tratados termicamente valores de coeficiente de determinação, entre o MOED e MOE, na faixa de 0,786 a 0,956 .

Souza (2009), verificando a avaliação não destrutiva de painéis LVL com a madeira de
Pinus oocarpa e Pinus kesiya, obteve $\mathrm{R}_{2}$ variando entre 0,476 e 0,586 , quando relacionou o MOEd com o MOE.

De forma geral, o presente trabalho, quando comparado com trabalhos encontrados na literatura, obteve altos valores de correlação entre o MOEd e MOE para os painéis de bagaço de cana.

\section{CONCLUSÕES}

Observou-se alta correlação de Pearson entre o MOEd e o MOE (0,770 a 0,986), além de elevados valores de coeficiente de determinação $\left(\mathrm{R}^{2}\right.$ de 0,594 a 0,976), para todos os tratamentos avaliados.

Quanto ao efeito das variáveis de produção, o tipo de material utilizado é o que exerce maior influência sobre a determinação do MOEd, sendo o material bagaço de cana o que apresentou as melhores correlações. O tipo de adesivo não apresentou influência sobre as correlações, enquanto que os painéis com estrutura em camadas tiveram tendência de melhoras das correlações em relação aos painéis homogêneos.

\section{AGRADECIMENTOS}

À Fundação de Amparo à Pesquisa do Estado de Minas Gerais (FAPEMIG), à Coordenação de Aperfeiçoamento de Pessoal de Nível Superior (CAPES) e ao Conselho Nacional de Desenvolvimento Científico e Tecnológico (CNPq).

\section{STATUS DA SUBMISSÃO}

Recebido: 23/08/2011

Aceito: 07/02/2012

Resumo publicado online: 22/03/2012

Artigo completo publicado: 30/06/2012

\section{AUTOR(ES) PARA CORRESPONDÊNCIA}

\section{Rafael Farinassi Mendes}

Departamento de Ciências Florestais - DCF,

Universidade Federal de Lavras - UFLA,

Campus Universitário,

CEP 37200-000, Lavras, MG, Brasil

e-mail: rafaelfarinassi@gmail.com 


\section{REFERENNCIAS}

Bodig J. The process of NDE research for Wood and Wood composites. The journal of nondestructive testing 2001; 6(3)

Del Menezzi CHS, Tomaselli I, Souza MR. Avaliação não-destrutiva de painéis OSB modificados termicamente: parte 1- efeito do tratamento térmico sobre a velocidade de propagação de ondas de tensão. Scientia Forestalis 2007; 76:67-75.

Deutsches Institut für Normung - DIN. Normen für Holzfaserplaten Spanplatten Sperrholz. DIN 52362: Testing of wood chipboards bending test, determination of bending strength. Berlin; 1982. p. 39-40.

Instituto Brasileiro de Geografia e Estatística - IBGE. Anuário estatístico do Brasil. Rio de Janeiro, 2010.

Mendes RF, Mendes LM, Guimarães Júnior JB, Mendonça LL. Qualidade de painéis aglomerados produzidos na China utilizando bagaço de cana. In: Anais do Encontro Brasileiro em Madeiras e em Estruturas de Madeira; 2008; Londrina. Londrina: Universidade Estadual de Londrina. 2008.
Mendes RF, Mendes LM, Guimarães Júnior JB, Santos, RC, Bufalino L. The adhesive effect on the properties of particleboards made from sugar cane generated in the distiller. Revista de Ciências Agrárias 2009; 32(2):209218.

Mendes RF, Mendes LM, Abranches RAS, Santos RCS, Guimarães Júnior JB. Painéis aglomerados produzidos com bagaço de cana em associação com madeira de eucalipto. Scientia Forestalis 2010; 38(86):285-295.

Morales EAM. Técnicas de propagação de ondas na estimativa de propriedades mecânicas de painéis OSB [tese]. São Carlos: Universidade de São Paulo; 2006.

Souza, F. Propriedades mecânicas, físicas, biológicas $e$ avaliação não-destrutiva de painéis de lâminas paralelas (LVL) com madeira de Pinus oocarpa e Pinus Kesiya [dissertação]. Brasília: Universidade de Brasília; 2009.

Vun RY, Wu Q, Monlezun CJ. Through-thickness ultrasonic characterization of wood and agricultural fibre composites. Forest Products Journal 2004; 54(12):233-239. 